\title{
Glyceryl trinitrate is a novel inhibitor of quorum sensing in Pseudomonas aeruginosa
}

\author{
Hisham A Abbas ${ }^{1}$, Moutaz A Shaldam²
}

1. Department of Microbiology and Immunology, Faculty of Pharmacy, Zagazig University, Zagazig, Egypt

2. Department of Medicinal Chemistry, Faculty of Pharmacy, Delta University for Science and Technology, Gamasa, Egypt

\begin{abstract}
Background: Targeting quorum sensing is an alternative approach to antibiotics.Targeting quorum sensing-regulated virulence will disarm the pathogen without exerting pressure on its growth. As a result, emergence of resistance is avoided and the immune system can easily eradicate bacteria.

Objectives: Investigation of the possible inhibition of quorum sensing-regulated virulence of Pseudomonas aeruginosa by glyceryltrinitrate.

Methods: The quorum sensing inhibiting activity of glyceryl trinitrate was assessed by inhibition of violacein production in Chromobacterium violaceum ATCC 12472. Its ability to inhibit pyocyanin, protease, biofilm and tolerance to oxidative stress was evaluated. Docking study was performed to study the interference of glyceryl trinitrate with the binding of autoinducers with LasR and rhlR receptors.

Results: Glyceryl trinitrate exerted a significant biofilm inhibiting and eradicating activities. It decreased the production of quorum-sensing dependent violacein production. It significantly inhibited the production of pyocyanin and protease and diminished the tolerance against oxidative stress. Molecular docking study showed that glyceryl trinitrate interferes with the binding of autoinducers to their receptors. It could bind to Las Rand rhlr receptors with binding energy of -93.47 and -77.23 , respectively. Conclusion: Glyceryl trinitrate can be an antivirulence agent in the treatment of Pseudomonas aeruginosa topical infections such as burn infections.
\end{abstract}

Keywords: Glyceryl trinitrate, Pseudomonas aeruginosa, quorum sensing, virulence inhibition.

DOI: http://dx.doi.org/10.4314/ahs.v16i4.29

Cite as: Abbas HA, Shaldam MA. Glyceryl trinitrate is a novel inbibitor of quorum sensing in Pseudomonas aeruginosa. Afri Health Sci. 2016;16(4): 1109-1117. bttp://dx.doi.org/10.4314/abs.v16i4.29

\section{Introduction}

Quorum sensing (QS) is a cell-to-cell communication system between bacterial cells ${ }^{1}$. It is based on the secretion of signaling molecules or autoinducers by the bacterial cells. Autoinducer molecules accumulate in the growth medium during bacterial growth and they reflect the population density. When a threshold concentration is reached, the expression of genes is changed. The genes of virulence and biofilm formation are controlled in many pathogenic bacteria by $\mathrm{QS}^{2}$. Autoinducers in many gram-negative bacteria are acyl homoserine lactones

\section{Corresponding author:}

Hisham Abdel Monem Abdel Hamid Abbas

Egypt, Zagazig, Zagazig University,

Faculty of Pharmacy,

Department of Microbiology and Immunology.

Fax No.: (002)0552303266

Telephone No: (002)01276112647

E-mail: hishamabbas2008@gmail.com

African Health Sciences Vol 16 Issue 4, December, 2016
(AHLs). The genes involved in AHL-based QS systems are referred to as luxI-like and luxR-like genes. The enzyme needed for the synthesis of an AHL is encoded by luxI-like gene, while the receptor of that specific AHL is encoded by the cognate luxR-like gene.

When the AHL signal is bound to the receptor, the transcription of target genes is regulated ${ }^{3} . P$. aeruginosa is an opportunistic pathogen that causes various infections, such as burn infections, especially in immuno suppressed patients ${ }^{4}$. QS enables $P$. aeruginosa to produce virulence factors involved in establishing infections. The virulence genes in $P$. aeruginosa are regulated by two AHL lux-like systems, lasI/lasR and rhlI/rhlR. In the lasI/lasR system, lasI synthesizes 3-oxo-dodecanoylhomoserine lactone (3-oxo-C12HSL) 5 . When exceeding a certain concentration, 3-oxo-C12HSL binds to the cytoplasmic receptor LasR and induces the expression of genes that produce virulence factors, such as proteases, elastases and exotoxin $\mathrm{A}^{6}$. Moreover, rhlI is regulated by LasR-3-oxo-C12HSL 
and it is responsible for the synthesis of butanoylhomoserine lactone (C4HSL). Butanoyl homo- serine lactone binds to the receptor RhlR and activates the genes encoding pyocyanin, elastases, siderophores, and rhamnolipids ${ }^{7}$. Biofilm formation in $P$. aeruginosa is also controlled by QS regulated factors such as rhamno- lipids and siderophores, and swarming motility. Bacteria growing in biofilm mode are present as different phenotypes from planktonic cells, so they are highly resistant to antibiotic therapy $^{8}$. The abuse of antibiotics in the treatment of burn wound infections led to the emergence of antibiotic-resistant microorganisms ${ }^{9}$. As a result, alternative therapeutic strategies are necessary. Due to the role of QS in regulation of virulence factors, it is an ideal target for novel anti-virulence drugs ${ }^{10}$.

Glyceryl trinitrate (GTN) is an agent used in the treatment of hypertension. However, it was found to possess wound healing and antimicrobial activities ${ }^{11,12}$.

The antibiofilm activity of GTN was previously reported. It was found to be an effective catherter lock solution in combination with ethanol and citrate ${ }^{13,14}$. Moreover, GTN combined with caprylic acid was able to eradicate biofilms of Staphylococcus aureus, Staphylococcus epidermidis, Pseudomonas aeruginosa and candida albicans ${ }^{14}$.

GTN exerted antimicrobial activity against planktonic Candida albicans at high concentrations ranging from $0.15 \%$ to $0.3 \%{ }^{12}$. GTN is approved from FDA to be used as an ointment for anal fissures with concentrations up to $0.4 \%{ }^{15}$.

The objective of this study was to investigate the ability of glyceryl trinitrate to inhibit QS-mediated virulence factors and biofilm formation in P. aeruginosa PAO1 strain and five clinical isolates from burn wound infections.

\section{Materials and methods}

\section{Media and chemicals}

Luria-Bertani (LB) broth, LB agar, tryptone and peptone were obtained from Lab M Limited (Lancashire, United Kingdom). Mueller Hinton broth, Mueller Hinton agar and Tryptone soya broth were the products of Oxoid (Hampshire, UK). Azocasein was purchased from Sigma (St. Louis, USA) and glyceryl trinitrate (Stock so- lution with a concentration of $1 \mathrm{mg} / \mathrm{ml}$ ) from POHL-Boskamp, Gmbh\&Co., Hohenlockstedt, Germany. Other chemicals were of pharmaceutical grade.

\section{Bacterial strains}

Pseudomonas aeruginosa PAO1 strain, Chromobacterium violaceum ATCC12472 and five clinical isolates obtained from patients with burn wound infections in Zagazig University hospitals were used in this study. The clinical isolates were obtained from the stock culture of the Microbiology and Immunology Department, Faculty of Pharmacy, Zagazig University. Pseudomonas aeruginosa PAO1 and Chromobacterium violaceum ATCC 12472 were purchased from ATCC organization, Manassas, Virginia, USA.

\section{Determination of minimum inhibitory concentra- tion (MIC)}

To determine the minimum inhibitory concentration of GTN, the agar dilution method was used according to the Clinical Laboratory and Standards Institute Guidelines $(\mathrm{CLSI})^{16}$. Overnight cultures of the tested strains were prepared in tryptone soya broth (TSB). The cultures were diluted with Mueller-Hinton broth to have turbidities matching that of $0.5 \mathrm{McF}$ arland Standard and then diluted 1:10 with sterile saline so that approximate celldensity of $10^{7} \mathrm{CFU} / \mathrm{ml}$ for each strain was achieved. A standardized inoculum was delivered to the surface of Mueller-Hinton agar plates with different GTN dilutions to achieve a final inoculum of approximately $10^{4} \mathrm{CFU}$ per spot on the agar. The plates were incubated at $37^{\circ} \mathrm{C}$ for $20 \mathrm{~h}$ and the MIC was calculated as the lowest concentration of the GTN that inhibited the visible growth in the wells.

\section{Violacein inhibition assay}

The effect of GTN on quorum sensing inhibition was investigated by testing the ability of GTN to inhibit quorum sensing-dependent violacein production in Chromobacterium violaceum ATCC 12472 according to Blosser and $\mathrm{Gray}^{17}$. C. violaceum was grown in LB broth, and after overnight incubation, the culture was diluted to achieve an approximate cell density of $10^{6} \mathrm{CFU} / \mathrm{ml}$. Aliquots of $100 \mu \mathrm{l}$ of the adjusted culture were used to inoculate 5 $\mathrm{ml}$ of LB broth in the absence and presence of GTN. After incubation at $30^{\circ} \mathrm{C}$ for $24 \mathrm{~h}$, aliquots of $100 \mu \mathrm{l}$ of sodium dodcyl sulphate $(10 \%)$ were added to aliquots of $200 \mu \mathrm{l}$ of each culture to lyse the cells. To extract violacein, water saturated butanol $(900 \mu \mathrm{l})$ was added followed by vortexing and centrifugation at 12,000 rpm for $5 \mathrm{~min}$ utes to separate the organic phase. The concentration of violacein in the organic phase was measured at $585 \mathrm{~nm}$ by microplate reader (Biotek, USA).

African Health Sciences Vol 16 Issue 4, December, 2016 


\section{Assay of biofilm formation inhibition and eradica- tion}

The ability of $P$. aeruginosa strains to produce biofilm was investigated by using the modified method of Stepanovic et al. ${ }^{18}$ Overnight cultures of the tested strains were used to prepare bacterial suspensions with densities of $1 \times 10^{6} \mathrm{CFU} / \mathrm{ml}$ using TSB for dilution. The bacterial suspensions were added in aliquots of $100 \mu$ l to the wells of 96 well sterile microtiter plates with rounded bottom. The plates were incubated for $24 \mathrm{~h}$ at $37^{\circ} \mathrm{C}$ and then the planktonic cells were removed by aspiration of the contents of the microtiter plates. The wells were washed 3 times with sterile phosphate buffered saline (PBS, $\mathrm{pH}$ 7.2) and aliquots of $100 \mu \mathrm{l}$ of $99 \%$ methanol were added to fix the attached bacteria and left for 20 minutes. The wells were stained using crystal violet (1\%) for 20 minutes and the wells were washed with distilled water to remove any excess dye.

The plates were left to air dry and the dye was eluted by addition of $33 \%$ glacial acetic acid. The contents of the microtiter plate were transferred to a new plate and the optical densities were measured with a spectrofluorimeter (Biotek, USA) at $590 \mathrm{~nm}$. Measurements were performed in triplicate. To identify the magnitude of biofilm formation, the cut-off optical density (ODc) was calculated as three times standard deviations above the mean OD of the negative control. According to the criteria of Stepanovic et al. ${ }^{18}$, the tested strains were either non-biofilm producer (OD $\leq \mathrm{ODc}$ ), weak biofilm producer (OD $>$ ODc, but $\leq 2 \mathrm{x}$ ODc), moderate biofilm producer $(\mathrm{OD}>2 \mathrm{x}$ ODc, but $\leq 4 \mathrm{x}$ ODc), or strong biofilm producer $(\mathrm{OD}>4 \mathrm{x} \mathrm{ODc})$.

To test the anti-biofilm activity of GTN, the previous procedure was followed in the absence and presence of $0.25 \mathrm{mg} / \mathrm{ml}$ of GTN. The percentage of biofilm inhibition was calculated by the following formula

$\%$ of biofilm inhibition $=(\mathrm{OD}$ in absence of GTN-OD in presence of GTN)/ OD in absence of GTN

To test the ability of GTN to eradicate established biofilms, $100 \mu$ l aliquots of GTN $(0.25 \mathrm{mg} / \mathrm{ml})$ were added to the wells of microtiter plates with preformed biofilms. The plates were incubated for $24 \mathrm{~h}$ at $37^{\circ} \mathrm{C}$ and treated as previously described in biofilm formation assay. The optical density was measured at $590 \mathrm{~nm}$ using spectrofluorimeter (Biotek, USA) and the percentage of biofilm eradication was calculated.

\section{Protease assay}

The proteolytic activity was determined by the azocaseinassay according to Kessleret al. ${ }^{19}$ Azocasein $(0.3 \%)$ was prepared in $0.05 \mathrm{M}$ Tris- $\mathrm{HCl}$ and $0.5 \mathrm{M} \mathrm{CaCl} 2(\mathrm{pH} 7.5)$ and $1 \mathrm{ml}$ was added to $150 \mu \mathrm{l}$ of the free cell culture supernatant. After incubation of the mixture for 15 minutes at $37^{\circ} \mathrm{C}, 0.5 \mathrm{ml}$ of trichloroacetic acid $(10 \%)$ was added to terminate the reaction. The precipitated azocasein was removed by centrifugation and the absorbance of the supernatants was measured at a wave length of $400 \mathrm{~nm}$ using Biotek Spectrofluorimeter (Biotek, USA).

\section{Pyocyanin assay}

The assay was performed according to Das and Manefield $^{20}$. The tested strains were grown overnight in LB broth and diluted to reach $\mathrm{OD}_{600}$ of 0.4 . Ten $\mu$ l of the diluted suspension of each strain was inoculated in $1 \mathrm{ml}$ of LB broth in the absence and presence of GTN $(0.25 \mathrm{mg} /$ $\mathrm{ml})$. The cultures were incubated at $37^{\circ} \mathrm{C}$ for $48 \mathrm{~h}$ and the tubes were centrifuged at 10,000 rpm for 10 minutes. The absorbance of pyocyanin in the supernatant was measured at $691 \mathrm{~nm}$ using Biotek Spectrofluorimeter (Biotek, USA). The experiment was performed in triplicate and the mean of the results and the percentage of pyocyanin inhibition were calculated.

\section{Sensitivity to oxidative stress}

The ability of GTN to interfere with resistance to oxidative stress mediated by pyocyanin was performed by a disk assay as modified from the method of Hassett et al. ${ }^{21}$ Aliquots of $100 \mu \mathrm{l}$ from overnight cultures of the tested bacterial strains in TSB were uniformly spread on the surface of TSA plates with $2 \%$ agar with and without GTN $(0.25 \mathrm{mg} / \mathrm{ml})$. Sterile filter paper disks of $6 \mathrm{~mm}$ diameter were put on the surface of TSA plates. The disks were loaded with $10 \mu \mathrm{l}$ of $1.5 \% \mathrm{H}_{2} \mathrm{O}_{2}$.

The plates were incubated at $37^{\circ} \mathrm{C}$ for $24 \mathrm{~h}$ and the diameters of the zones of inhibition were measured.

\section{Docking study}

The crystal structure of $P$. aeruginosa LasR ligand binding domain was retrieved from Protein Data Bank (PDB ID: 2UV0) ${ }^{22}$ and the rhlr receptor model (ID:P54292.1) was obtained from protein model portal (Protein Model Portal) ${ }^{23}$. This study was carried out on GTN along with 
the co-crystalized natural ligand, 3-oxo-dodecanoyl homoserine lactone, and the C30 furanone inhibitor into the receptor active site using Molegro Virtual Docker (MVD Version 6.0). GTN was drawn into Marvin Sketch V5.11.5, 2013.

The most energetically favored conformer was saved as (*.mol2) file format for docking. The optimal geometry of the ligand was determined during the docking process. The $\mathrm{E}$ monomer was chosen for analysis, water was removed, cavities were detected and the search area was set to be $9 \AA$ from the center of the active site. Iterative simplex algorithm was chosen to perform docking process with 10 runs per ligand, 200 population size, $1000 \max$ iteration and 5 poses for each ligand. MolDock docking engine using docking template and the optimized ligands was executed ${ }^{24}$. Finally, the top returned poses were chosen for analysis. The same docking procedures were fol- lowed for docking GTN and the autoinducer, butanoyl homoserine lactone(C4HSL), into the active site of the rhlr receptor model.

\section{Statistical analysis}

The effects of GTN on virulence factors and biofilm formation and eradication in P. aeruginosa were compared by One Way ANOVA followed by Dunnett's Multiple Comparison Test, Graph Pad Prism 5. The effect on oxidative stress and violacein production was analyzed using unpaired t test. $P$ values $<0.05$ were considered statistically significant.

\section{Results}

\section{Biofilm formation assay}

Strong biofilm formation capacity was found with clinical strains as the OD was higher than 4ODc (0.272) (Table 1). PAO1 strain is a well known strong biofilm forming strain.

Table 1. Biofilm assay of Pseudomonas aeruginosa strains

\begin{tabular}{|cc|}
\hline Bacterial strain & OD590 (mean \pm SD) \\
\hline "PA1 & $0.773 \pm 0.065$ \\
PA2 & $1.037 \pm 0.0390$ \\
PA3 & $0.618 \pm 0.084$ \\
PA4 & $0.628 \pm 0.031$ \\
PA5 & $0.627 \pm 0.059$ \\
\hline
\end{tabular}

Biofilm inhibition and eradication activities of GTN Sub-inhibitory concentration of GTN $(0.25 \mathrm{mg} / \mathrm{ml}$ that corresponds to atleast $1 / 4$ MIC) showed significant biofilm inhibiting activity against tested strains $(p<0.05)$. The inhibition ranged between $61 \%$ with PAO1 strain and $92.26 \%$ with clinical isolate PA4. The biofilm eradicating activity was lower but still significant $(\mathrm{p}<0.05)$ (Fig. 1). GTN biofilm removal capacity ranged between $28.51 \%$ and $66.52 \%$ with PA3 strain and PAO1, respectively.
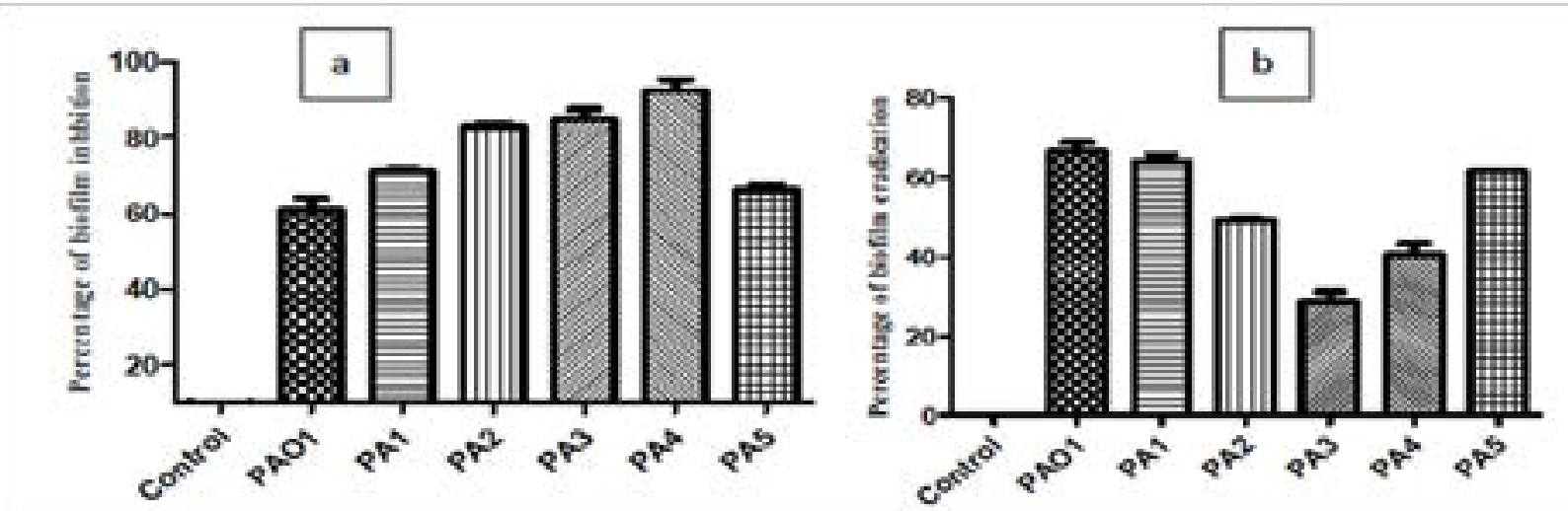

Figure 1.(a) Inhibition of biofilm formation by GTN, (b) eradication of established biofilms by GTN. The error bars indicate the standard deviations of two measurements 
Inhibition of violacein production

The effect of GTN on inhibition of quorum sensingdependent violacein production in the biosensor strain Chromobacterium violaceum ATCC 12472 was statistically significant $(\mathrm{p}<0.05)$. GTN could inhibit violacein production by $70.08 \%$.
This confirms the anti-quorum sensing activity of GTN.

\section{Protease inhibition assay}

GTN significantly reduced protease production $(\mathrm{p}<0.05)$. The percentage of inhibition ranged between 52.87\% with PA3 and 78.53\% with PAO1 (Fig. 2).

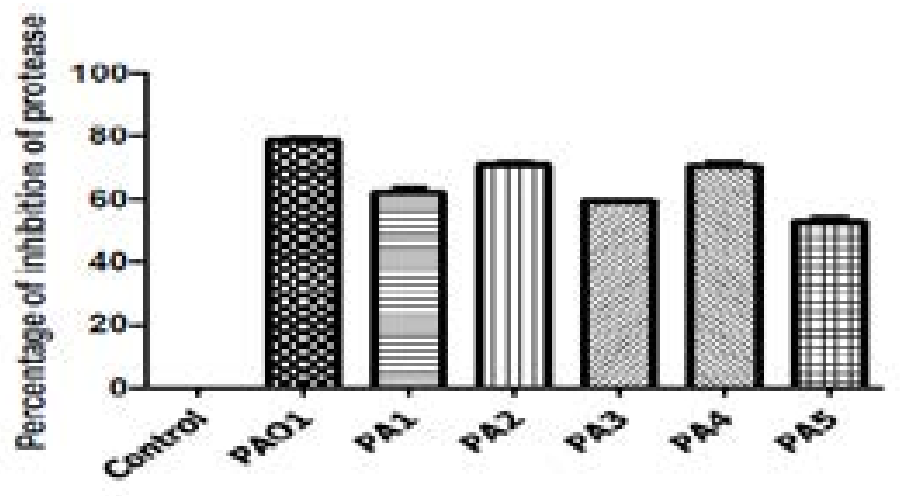

Figure 2. Inhibition of protease by GTN. The error bars indicate the standard deviations of three measurements

Pyocyanin inhibition assay

Pyocyanin inhibition by GTN was statistically signifi- $74.93 \%$ with PAO1 (Fig. 3).

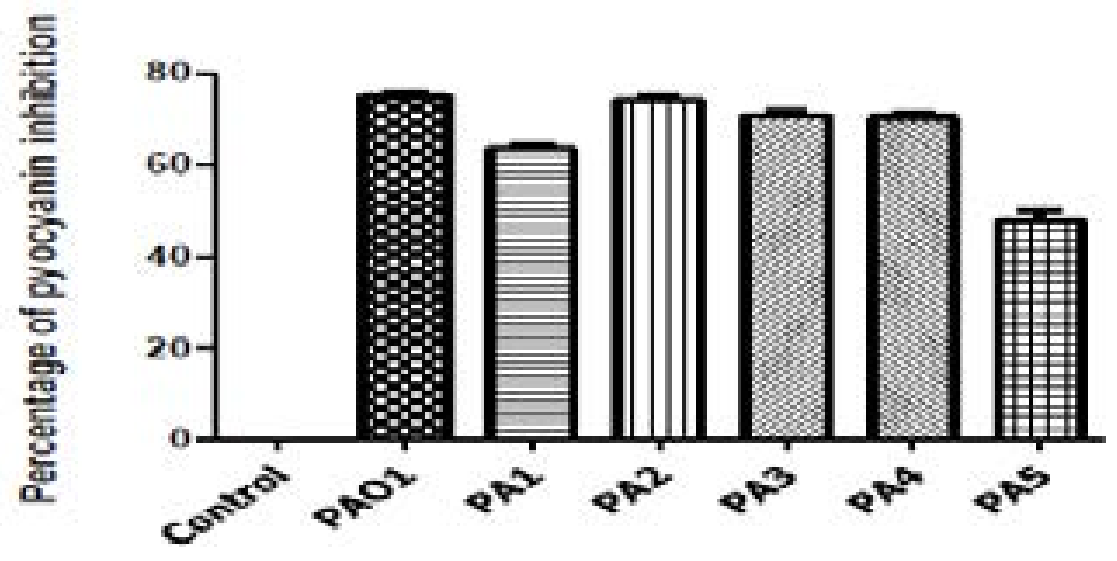

Figure 3. Inhibition of pyocyanin production by GTN. The error bars indicate the standard deviations of three measurements 
Oxidative stress assay

GTN was found to significantly increase the inhibitory effect of hydrogen peroxide $(\mathrm{p}<0.05)$ (Fig. 4). The diameters of inhibition zones were $26 \pm 2 \mathrm{~mm}$ (mean $\pm \mathrm{SD}$ ) and $40 \pm 2$ in the absence and presence of GTN, respectively.
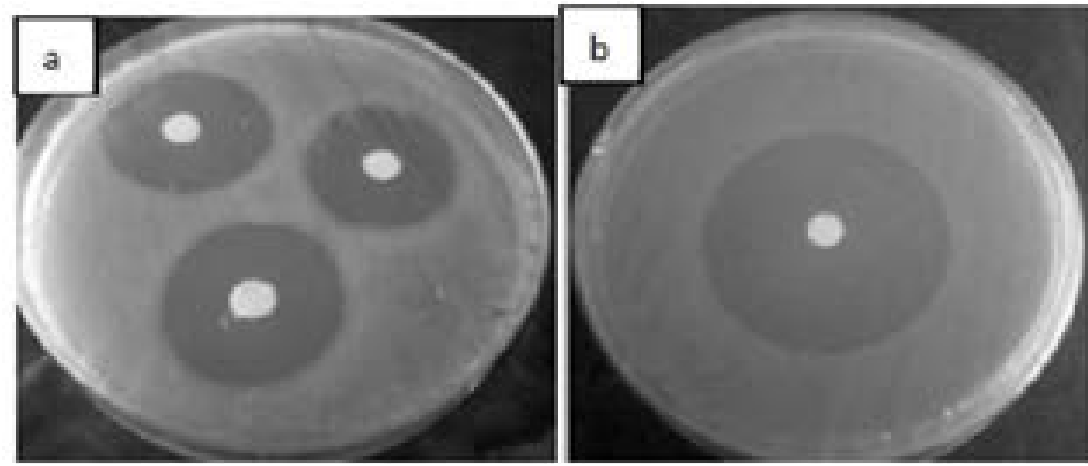

Figure 4. Resistance of Pseudomonas aeruginosa PAO1 strain to oxidative stress in absence (a) and presence (b) of GTN. The zone of inhibition of growth of PAO1 by hydrogen peroxide increased in the presence of GTN

\section{Docking study}

Molecular docking study was anticipated to interpret the mode of binding interaction of the GTN to the quorum sensing proteins LasR and rhlr at the molecular level. The natural ligand, GTN and the inhibitor C30 furanone new were docked into LasR active site. The binding interaction between GTN and the receptor is attributed to Hbonding with Tyr56, Trp60, Thr75, Tyr93, Thr115 and Ser129 (Fig. 5). The C30 furanone, a known inhibitor to LasR, was found to bind through $\mathrm{H}$ - bond with $\operatorname{Trp} 60$ and Arg 61, while the natural ligand makes H-bond with Tyr56, Trp60, Asp73 and Ser129. The MolDock score of GTN, C30 furanone and the natural ligand were $-93.472,-80.468$ and -164.498 . The inhibitor is unable to induce the correct formation of the hydrophobic core of LasR. This conformational change is important for LasR activity $^{22}$. The natural ligand is a LasR inducer as it can stabilize this conformational change through its long acyl chain, while GTN and C30 destabilize the protein core. Thus GTN and C30 furanone produce LasR antagonistic activity.
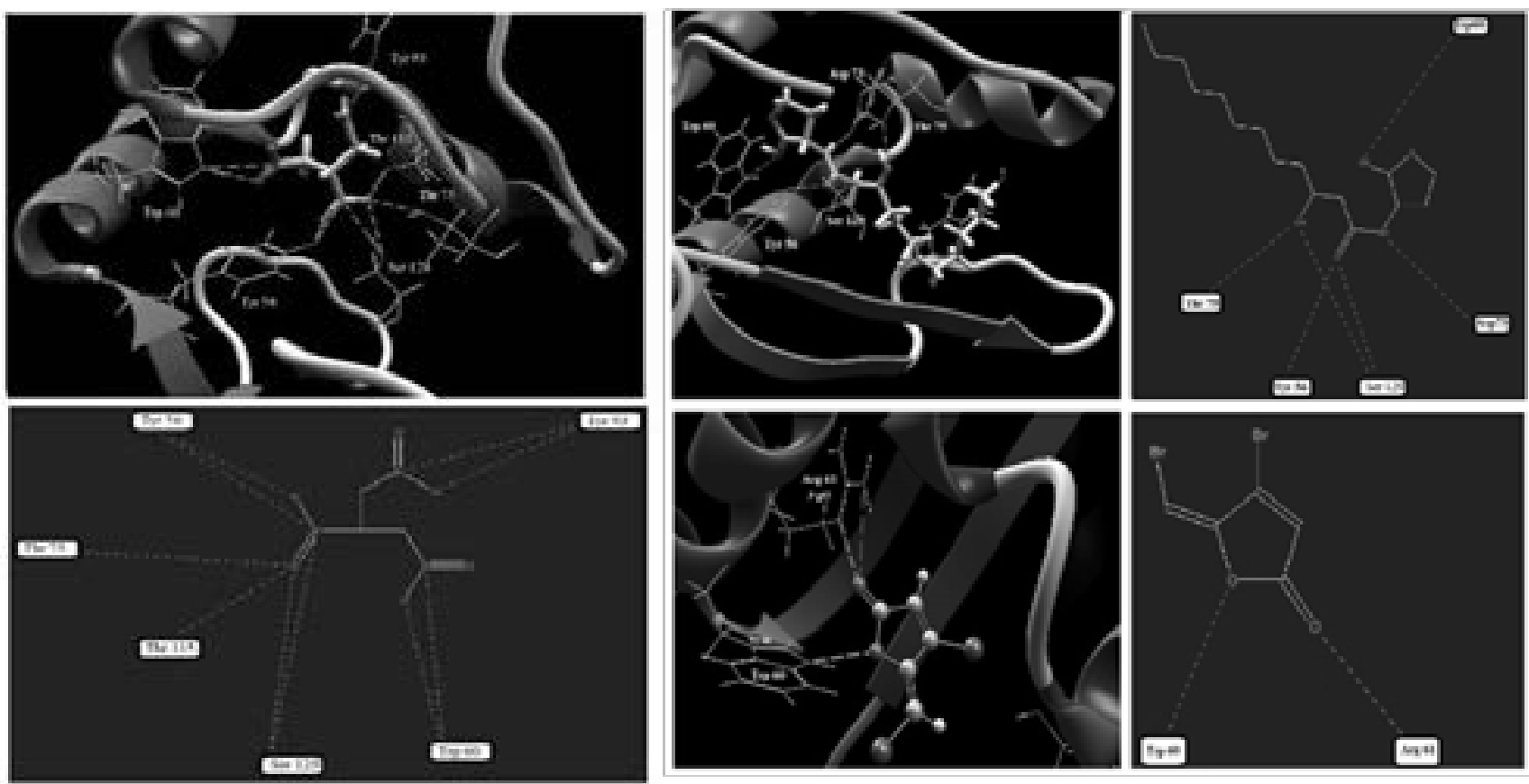

Figure 5. The Molecular docking of a) GTN into the active site of LasR enzyme 3D (Top) and 2D schematic view of the binding (Bottom), b) natural ligand (Top) and C30 furanone (Bottom) into the active site of LasR enzyme 3D (Left) and 2D schematic view of the binding (Right) 
Docking of GTN into rhlr receptor model active site showed similar interaction to the auoinducer C4HSL (Fig. 6). Both make H-bond with Trp108 and Arg1 ${ }^{12}$. C4HSL shows an extra H-bond with the carbonyl backbone of
Leu ${ }^{12}$. These interactions resulted in binding energy of -77.23 and -85.24 for GTN and C4HSL respectively. The acyl group of C4HSL is an important factor for an inducer that is absent in GTN which makes it a possible inhibitor.

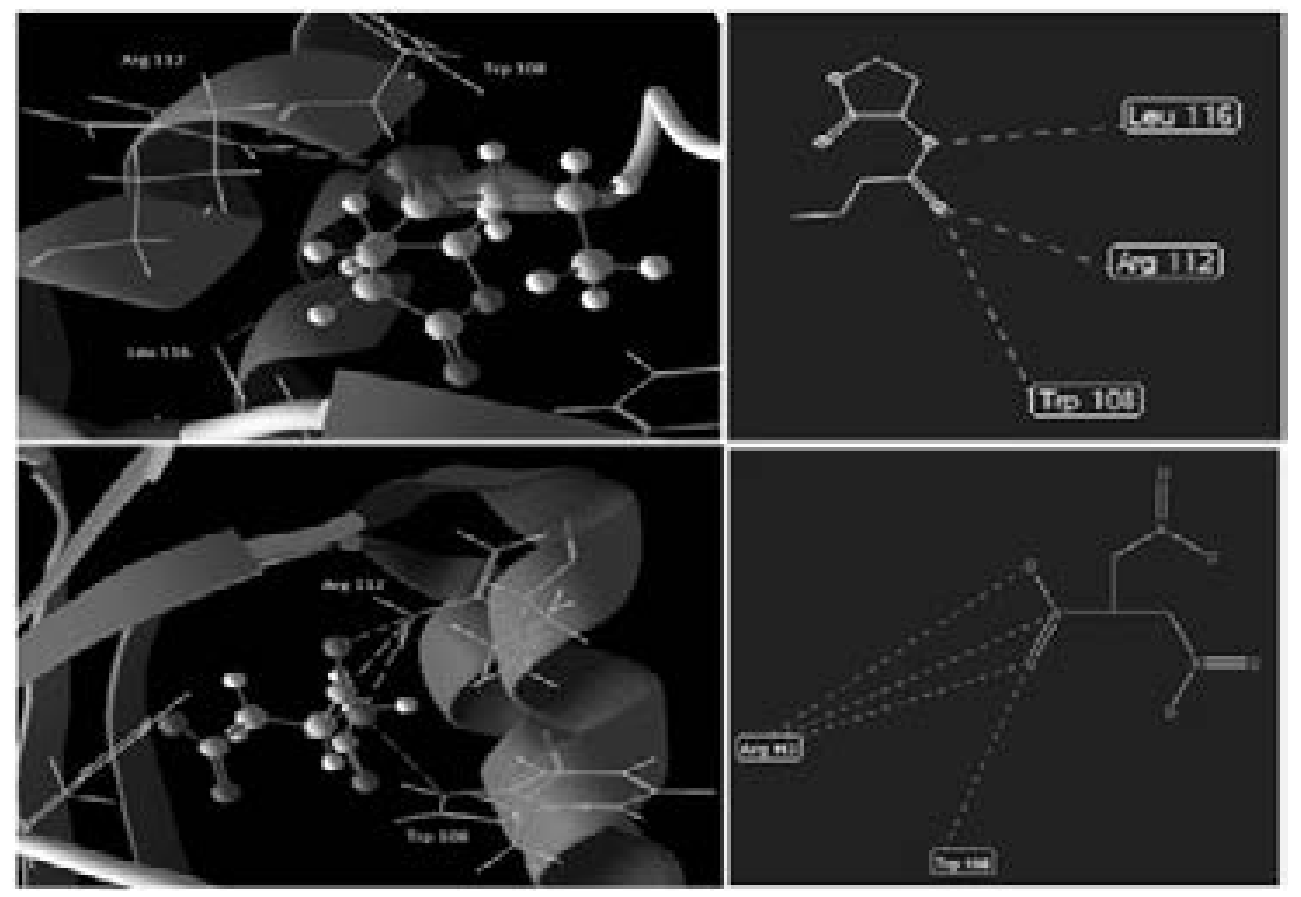

Figure 6. The Molecular docking of C4-BHL (Top) and GTN (Bottom) into the active site of rhlr receptor model 3D (Left) and 2D schematic view of the binding (Right)

\section{Discussion}

The role of quorum sensing in the control of the virulence made it an attractive target for new anti-virulence agents that can be used to treat infections. Many studies on QS inhibition in Gram negative bacteria tested the ability of structural homologues of QS autoinducers to interfere with binding of these autoinducers to their receptors resulting in QS inhibition ${ }^{25}$.

The high toxicity of most QSIs hinders their use in the treatment of infections ${ }^{25}$. As a result, it is beneficial to search for new QSIs among FDA approved drugs that makes their clinical application possible. This rationale has been used in previous studies. As a result, a number of useful quorum sensing inhibitors have been found among FDA approved drugs such as niclosamide, aspirin and diclofenac sodium ${ }^{26-28}$.

In this study, glyceryl trinitrate at a sub-inhibitory concentration was investigated for its activity against quorum sensing and some of QS-regulated virulence factors such as pyocyanin, protease in addition to tolerance to oxidative stress and biofilm formation.

African Health Sciences Vol 16 Issue 4, December, 2016
GTN could inhibit the violacein pigment production that is under quorum-sensing control in the reporter strain Chromobacterium violaceum ATCC 12472. This constitutes the biological evidence of the quorum sensing inhibiting activity of GTN. Moreover, molecular docking study showed that GTN is a competitive inhibitor of the natural ligands of LasR and rhlR receptors; namely 3-oxoC12-HSL and C4-HSL, respectively. Consequently, GTN could inhibit the binding of these autoinducers to their receptors resulting in quorum sensing inhibition. GTN showed higher quorum sensing inhibiting activity than the classical quorum sensing inhibition C30 furanone ${ }^{29}$ as competitive inhibitors of 3-oxo-C12- HSL as shown by lower docking score of GTN than that of C30 furanone.

It also showed strong inhibiting activities against some QS-controlled virulence factors such as pyocyanin and protease production. Moreover, it could inhibit and eradicate the established biofilms. This is an additional proof of the anti-QS activity of GTN due to the involvement of QS system in biofilm formation and development ${ }^{30}$. GTN significantly reduced the tolerance of Pseudomonas aeruginosa PAO1 strain to oxidative stress. This may be 
due to the inhibition of QS that is directly related to the tolerance to oxidative stress in $P$. aeruginosa. QS enables $P$. aeruginosa to inhibit phagocytosis and intracellular killing by oxygen radicals in neutrophils ${ }^{31}$. Moreover, QSdeficient mutants produce lower amounts of catalase and superoxide dismutase and they are more susceptible to oxidative stress than the wild strain ${ }^{32}$.

GTN is safe for topical application, and even if systemic absorption occurs, GTN has a very short half-life (1 to 3 min), so any systemic effects are transient. Bolus doses of $200 \mathrm{~g}$ of GTN have been well tolerated and had no clinically significant adverse effects ${ }^{33-36}$.

\section{Conclusion}

GTN is a novel QSI and antivirulence drug that is approved by FDA for human use. It can be used topically for treatment of the highly resistant Pseudomonas aeruginosa topical infections such as burn infections.

\section{Conflict of interest}

The authors declare that they have no conflict of interest.

\section{References}

1. Fuqua WC, Winans SC, Greenberg EP. Quorum sensing in bacteria: the Lux R- Lux I family of cell density responsive transcriptional regulators. Journal of Bacteriology 1994; 176: 269-275.

2. Rutherford ST, Bassler BL. Bacterial Quorum Sensing: its role in virulence and possibilties of its control. Cold Spring Harbor Prespectives in Medicine 2012; 2:a012427.

3. Waters CM, BasslerLB.QuorumSensing:Cell-to-Cell Communication in Bacteria. Annual Review of Cell and Developmental Biology 2005; 21:319-346.

4. LyczakJB, Cannon CL, Pier GB. Lung infections associated with cystic fibrosis. Clinical Microbiology Reviews 2002; 15:194-222.

5. ParsekMR, Val DL, Hanzelka BL, Val DL, Hanzelka BL, CronanJr JE, Greenberg EP. Acyl homoserine lactone quorum sensing signal generation. Proceedings of the National Academy of Sciences of the United States of America 1999; 96:4360-4365.

6. Parsador L, Cook JM, GambelloMJ, Rust L, Iglewski BH. Expression of Pseudomonas aeruginosa virulence genes requires cell-to-cell communication. Science 1993; 260:1127-1130.

7. Wagner VE, Filiatrault MJ, Picardo KF, Iglewski BH. Pseudomonas aeruginosa virulence and pathogenesis issues.
In: Cornelis P (ed) Pseudomonas genomics and molecular biology, 1stedn. Caister Academic Press, Norfolk, UK, 2008; pp 129-158.

8. Costerton JW, Lewandowski Z, Caldwell DE, Korber DR, Lappin-Scott HM. Microbial Biofilms. Annual Review of Microbiology 1995; 49: 711- 745.

9. Fischbach MA, Walsh CT. Antibiotics for emergingpathogens. Science 2009; 325:1089-1093

10. Marshall J. Quorum sensing. Proceedings of the National Academy of Sciences of the United States of America 2013; 110:8.

11. Gorfine SR. Treatment of benign anal disease with topical nitroglycerin. Dis Colon Rectum 1995; 38: 453-457. 12. Palmeira-de-Oliveira A, Ramos AR, Gaspar C, Palmeira-de-Oliveira R, GouveiaP, Martinez- de-Oliveira J. In vitro anti-Candida activity of lidocaine and nitroglycerin: alone and combined. Infectious Diseases in Obstetrics and Gynecology 2012; 2012: Article ID 727248, 4pages.

13. Rosenblatt J, Reitzel R, Dvorak T, JiangY, Hachem R, Raad I. Glyceryl trinitrate complements citrate and ethanol in a novel antimicrobial catheter lock solution to eradicate biofilm organisms. Antimicrobial Agents and Chemotherapy 2013; 57:3555-3560.

14. Rosenblatt J, Reitzel RA, Raad I. Caprylic acid and glyceryl trinitrate combination for eradication of biofilm. Antimicrobial Agents and Chemotherapy 2015; 59:1786-1788. 15. Fenton C, Wellington K, Easthope SE. 0.4\% nitroglycerin ointment: in the treatment of chronic anal fissure pain. Drugs 2006; 66:343-349.

16. Clinical and Laboratory Standards Institute. Methods for dilution antimicrobial susceptibility tests for bacteria that grow aerobically; Approved standard, CLSI Document M07-A9, Vol. 32, No. 3. Wayne, PA, USA, 2012.

17. Blosser RS, Gray KM. Extraction of violacein from Chromo bacterium violaceum provides a new quantitative bioassay for $\mathrm{N}$-acyl homoserine lactone auto inducers. Journal of Microbiological Methods 2000; 40:47-55.

18. Stepanovic S, Vukovic D, Hola V, Di Bonaventura G, DjukićS, Ćirković I, Ruzicka F. Quantification of biofilm in microtiter plates: overview of testing conditions and practical recommendations for assessment of biofilm production by staphylococci. APMIS 2007; 115(8): 891-899.

19. Kessler E, Safrin M, Olson JC, Ohman DE. Secreted LasA of Pseudomonas aeruginosa is a staphylolytic protease. Journal of Biological Chemistry 1993; 268(10): 7503-7508.

20. Das T, Manefield M. Pyocyanin promotes extracellular DNA release in Pseudomonas aeruginosa. PLoS One 2012; 7:e46718

African Health Sciences Vol 16 Issue 4, December, 2016 
21. Hassett DJ, SchweizerHP, Ohman DE. Pseudomonas aeruginosa sodA and sodB mutants defective in manganeseand iron cofactored superoxide dismutase activity demonstrate the importance of the iron-cofactoredform in aerobic metabolism. Journal of Bacteriology 1995; 177:63306337.

22. Bottomley MJ, Muraglia E, Bazzo R, Carfi A. Molecular insights into quorum sensing in the human pathogen Psendomonas aeruginosa from the structure of the virulence regulator LasR bound to its autoinducer. Journal of Biological Chemistry 2007; 282:13592-13600.

23. www.proteinmodelportal.org/?pid=modelDetail\&p rovider $=$ SWISSMOD-EL\&template $=41$ fuA\&pmpuid $=$ $1000801294232 \&$ range_from $=1 \&$ range_to $=241 \&$ ref ac $=$ P54292\&mapped_ac $=$ P54292\&zid=async.

24. Thomsen R, Christensen MH. MolDock: A New Technique for High-Accuracy Molecular Docking. Journal of Medicinal Chemistry 2006; 49: 3315-3321.

25. Galloway WR, Hodgkinson JT, Bowden S, Welch M, Spring DR. Applications of small molecule activators and inhibitors of quorum sensing in Gram-negative bacteria. Trends in Microbiology 2012; 20:449-458.

26. Imperi F, Massai F, PillaiCR, Longo F, Zenn- aro E, RampioniG, ViscaP, LeoniL. Newlifefor an old drug: the anthelmintic drug niclosamide inhibits Pseudomonas aeruginosa quorum sensing. Antimicrobial Agents and Chemotherapy 2013; 57(2):996-1005.

27. El-Mowafy SA, Abd El Galil KH, El-Mess- erySM, Shaaban MI. Aspirin is an efficient in- hibitorof quorum sensing, virulence and toxins in Pseudomonas aeruginosa. Microbial Pathogenesis 2014; 74: 25e32.

28. Abbas HA (2015) Inhibition of virulence factors of Pseudomonas aeruginosa by diclofenac sodium. Roumanian Archives of Microbiology and Immunology 2015; 74(34):79-85.
29. DeNys R, Givskov M, Kumar N, KjellebergS, and Steinberg PD. Furanones. Progress in Molecular and Subcellular Biology 2006; 42:55-86.

30. Kirisits MJ, Parsek MR. Does Pseudomonas aeruginosa use intercellular signalling to build biofilm communities? Cell Microbiology 2006; 8: 1841-1849.

31. Hassett DJ, Ma JF, Elkins JG, McDermott TR, Ochsner UA, West SE et al. Quorum sensing in Pseudomonas aeruginosa controls expression of catalase and superoxidedismutasegenes and mediates biofilm susceptibility tohydrogenperoxide. Molecular Microbiology 1999; 34:10821093.

32. Bjarnsholt T, Jensen PO, Burmolle M, Hentzer M, Haagensen JA, Hougen $\mathrm{HP}$ et al. Pseudomonas aeruginosa tolerance to tobramycin, hydrogen peroxide and polymorpho nuclear leukocytes is quorum-sensing dependent. Microbiology 2005; 151:373-38.

33. Hill NS, Antman EM, Green LH, Alpert JS. Intravenous nitroglycerin. A review of pharmacology, indications, therapeutic effects and complications. Chest 1981;79:69-76.

34. Zalenski RJ, Levy P, Compton S, Delgado G, Welch $\mathrm{R}$, Waselewsky D. The feasibility of treating severe acute congestive heart failure with bolus intravenous nitroglycerin. Annals of Emergency Medicine 2004; 44: S134-S135.

35. Levy P, Compton S, Welch R, Delgado G, Jennett A, Penugonda N. Treatment of severe decompensated heart failure with high-dose intravenous nitroglycerin: a feasibility and outcome analysis. Annals of Emergency Medicine 2007; 50: 144-152.

36. Pullen KM, Riley ET, Waller SA, Taylor L, Caughey $\mathrm{AB}$, Druzin ML. Randomized comparison of intravenous terbutalinevs nitroglycerin for acute intrapartumfetal resuscitation. American Journal of Obstetrics and Gynecology 2007; 197:414.e1-414.e6. 